\title{
An Adaptively Segmented Forward Problem Based Non-Blind Deconvolution Technique for Analyzing SRAM Margin Variation Effects
}

\author{
Worawit Somha and Hiroyuki Yamauchi
}

\begin{abstract}
This paper proposes an abnormal Vshaped-error-free non-blind deconvolution technique featuring an adaptively segmented forward-problem based iterative deconvolution (ASDCN) process. Unlike the algebraic based inverse operations, this eliminates any operations of differential and division by zero to successfully circumvent the issue on the abnormal V-shaped error. This effectiveness has been demonstrated for the first time with applying to a real analysis for the effects of the Random Telegraph Noise (RTN) and/or Random Dopant Fluctuation (RDF) on the overall SRAM margin variations. It has been shown that the proposed ASDCN technique can reduce its relative errors of RTN deconvolution by $10^{13}$ to $10^{15}$ fold, which are good enough for avoiding the abnormal ringing errors in the RTN deconvolution process. This enables to suppress the cdf error of the convolution of the RTN with the RDF (i.e., fail-bit-count error) to $1 / 10^{10}$ error for the conventional algorithm.
\end{abstract}

Index Terms-Non blind deconvolution, SRAM, random telegraph noise, time-dependent margin variation

\section{INTRODUCTION}

The guard band (GB) design for static random access memory (SRAM) is expected to become a critical challenge because the increased time-dependent (TD) margin variations (MV)-caused failures cannot be predicated any more by only the convolution analyses $[1$, 5]. This is because (1) TD-MV, (i.e., unknown MV after being shipped to the market), will become much larger than the non-TD-MV, (i.e., given MV based on the measurements), resulting in the TD-MV dominating over the overall MV. This leads to a rapid increase in pressure to figure out the unknown factors by solving the inverse problem [6, 8], even though SRAM designers are unfamiliar with such a methodology and (2) the tail distribution of the convolution results of the TD-MV and non-TD-MV no longer shows Gaussian behavior but more complex mixtures of Gamma distributions [3-7], as shown in Figs. 1(a) and (b). Traditional SRAM statistical analyses including its convolution integral have no choice but to rely on the simple Gaussian model. Its parameters are extracted in advance using the measured data. On the other hand, if the non-Gaussian unknown factors no longer account for just a fraction but a large percentage of the overall MV(shown in Fig. 1(b)), the non-Gaussian inverse problem needs to be solved based on the pre-defined hypothesis of the unknown factors or final target specifications, as shown in Figs. 1(c) and (d). To clarify the challenges behind the proposed ideas in this paper, the concepts of what will be crucial in the coming process generations are shown in this section.

First of all, the relationship between the threshold

\footnotetext{
Manuscript received Jan. 24, 2014; accepted Jun. 10, 2014 A part of this work was presented in International SoC Design Conference (ISOCC), Busan in Korea, November 2013.

Fukuoka Institute of Technology, Japan

E-mail : yamauchi@fit.ac.jp
} 
voltage (Vth) and random dopant fluctuation (RDF) and the random telegraph noise (RTN) needs to be examined. References [4-6] reported that the distributions for the Vth variation $(\sigma \mathrm{Vth})$ due to the $\mathrm{RDF}$ and the RTN, i.e. $\sigma$ Vth_RDF and $\sigma$ Vth_RTN, obeyed the Gaussian and the Gamma distributions, respectively. "RTN distribution" and "RDF distribution", which are used in this paper, represent the Vth distributions with $\sigma$ Vth_RTN and $\sigma \mathrm{Vth} \_\mathrm{RDF}$, respectively.

Therefore, the Vth random variation $(\sigma \mathrm{V}$ th) considering both the RDF and RTN effects can be obtained from the convolution of $\sigma \mathrm{Vth} \_\mathrm{RDF}(f(x))$ with $\sigma$ Vth_RTN $(\boldsymbol{g}(\boldsymbol{x}))$. As a result, the distribution of the entire $\sigma \mathrm{Vth}(\boldsymbol{h}(\boldsymbol{x}))$ can be expressed as

$$
h(x)=f(x) \otimes g(x)
$$

In Fig. 1, the probability of the density function (pdf) of $\boldsymbol{f}(\boldsymbol{x})$ and $\boldsymbol{g}(\boldsymbol{x})$ for $\sigma \mathrm{Vth}$ tRDF and $\sigma \mathrm{Vth}$ tRTN were assumed to obey the Gaussian and Gamma distributions that are given by the NORMSDIST(x) and GAMMA.DIST(x, $\sigma, \beta)$ in the Microsoft Excel function, where $\sigma$ and $\beta$ are the shape and inverse scale parameters, respectively.

" $\mathrm{x}$ " is used as a title for the $\mathrm{x}$-axis in Fig. 1, and represents the scale of the distributions of the random variation, such as $\sigma \mathrm{Vth} \_\mathrm{RDF}$ and $\sigma \mathrm{Vth}$ RTN. The $\mathrm{x}$-axis was normalized to address the different Gaussian distributions for $\sigma \mathrm{Vth}$ RDF and the Gamma distributions for $\sigma$ Vth_RTN in the same figure, as shown in Fig. 2(b). In this paper, $\mu$ and $\sigma$ for the Gaussian RDF distribution are assumed $\mu=1$ and $\sigma=1$. The relationship between the probability density function (PDF) and the $\mathrm{x}$ (raw score) is shown in Fig. 2(b). On the other hand, the parameter $\operatorname{set}(\alpha, \beta)$ for 3-different Gamma distributions of RTN-1, RTN-2, and RTN-3 are assumed as(1, 0.05), (1, $0.25)$ and $(1,0.56)$, which determine the relationship of the tail length at $P D F=10^{-12}$ between the RDF, RTN-1, RTN-2, and RTN-3, as shown in Fig. 2(b).

The "z-score" can be used to normalize the x-axis only for a Gaussian distribution, which is expressed as (2)

$$
\mathrm{z}=(\mathrm{x}-\mu) / \sigma
$$

where $\mu$ and $\sigma$ are the parameters for the mean and standard deviation, respectively.
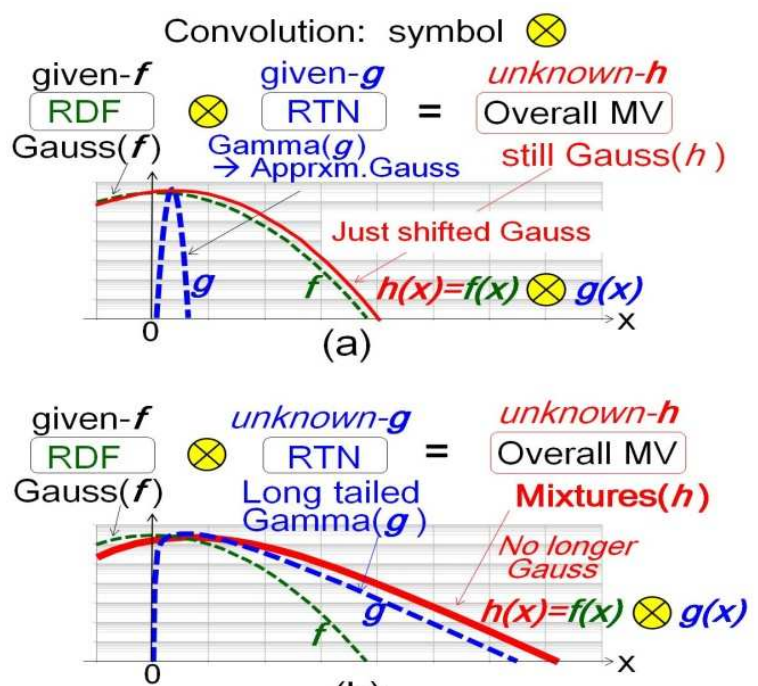

(b)

De-convolution: symbol $\otimes^{-1}$

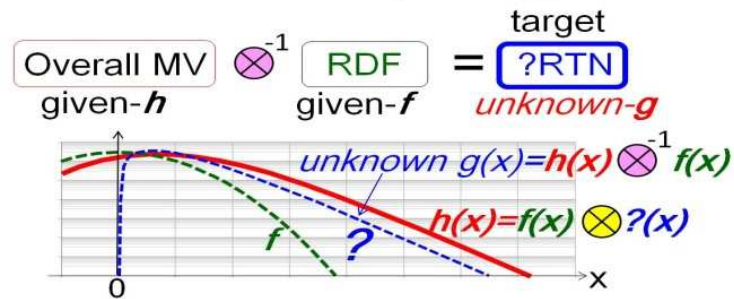

(c)

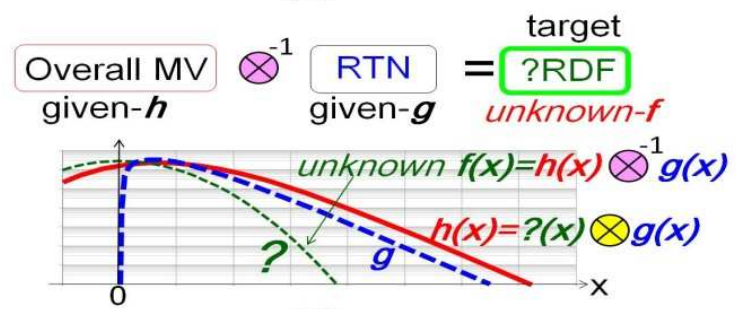

(d)

Fig. 1. Convolution of RDF with RTN (a) traditional, (b) new relationship between RDF, RTN. Extraction of (c) RTN, (d) RDF from the overall MV with (c) RDF, (d) RTN based on the deconvolution.

On the other hand, the "z-score" cannot be used for the Gamma distribution. Therefore, "x (raw score)" was used as a title for the $\mathrm{x}$-axis for both $\sigma \mathrm{Vth} \_\mathrm{RDF}$ and $\sigma$ Vth_RTN. Since $\mu=1$ and $\sigma=1$ are assumed in this paper, "z-score" = "x (raw score)".

The relationship between the $\sigma \mathrm{Vth}$ and $\mathrm{MV}$, such as random variations of the static noise margin $(\sigma \mathrm{SNM})$ or $\mathrm{V}_{\mathrm{DD} \text {,min }}\left(\sigma \mathrm{V}_{\mathrm{DD}, \text { min }}\right)$ caused by $\sigma \mathrm{Vth}$, were assumed for simplicity [8] in this paper as follows.

$$
\begin{gathered}
\sigma \mathrm{SNM}(\mathrm{x})=\mathrm{k} 1 \times \sigma \mathrm{V} \operatorname{th}(\mathrm{x}) \\
\sigma \mathrm{V}_{\mathrm{DD}, \min }(\mathrm{x})=\mathrm{k} 2 \times \sigma \mathrm{Vth}(\mathrm{x})
\end{gathered}
$$




$$
\begin{gathered}
\mathrm{SNM}(\mathrm{x})=\mathrm{SNM} \text { _mean }+\sigma \mathrm{SNM}(\mathrm{x}) \\
\mathrm{V}_{\mathrm{DD} \text {,min }}(\mathrm{x})=\mathrm{V}_{\mathrm{DD} \text {,min } \_ \text {mean }}+\sigma \mathrm{V}_{\mathrm{DD} \text {,min }}(\mathrm{x})
\end{gathered}
$$

where $\mathrm{k} 1$ and $\mathrm{k} 2$ represent a linear correlation coefficient [8]. SNM_mean and $\mathrm{V}_{\mathrm{DD} \text {,min_mean }}$ are the values of $\mathrm{SNM}$ and $\mathrm{V}_{\mathrm{DD} \text {,min }}$ excluding the $\sigma \mathrm{V}$ th effects on the MV. In this paper, SNM_mean and $\mathrm{V}_{\mathrm{DD} \text {,min_mean were }}$ assumed to be 0 so only the $\sigma \mathrm{V}$ th effects on the MV are discussed, as shown in Fig. 1.

The increasing paces of the $\sigma \mathrm{Vth}$ amplitude are differently dependent on the MOSFET size scaling parameter like the expressions in (7) and (8) below.

$$
\begin{aligned}
& \Delta \mathrm{Vth}(\mathrm{RDF}) \propto \mathrm{AVt}(\mathrm{RDF}) / \sqrt{\mathrm{LW}} \\
& \Delta \mathrm{Vth}(\mathrm{RTN}) \propto \mathrm{AVt}(\mathrm{RTN}) / \mathrm{LW}
\end{aligned}
$$

where AVt is the Pelgrom coefficient and the LW are the

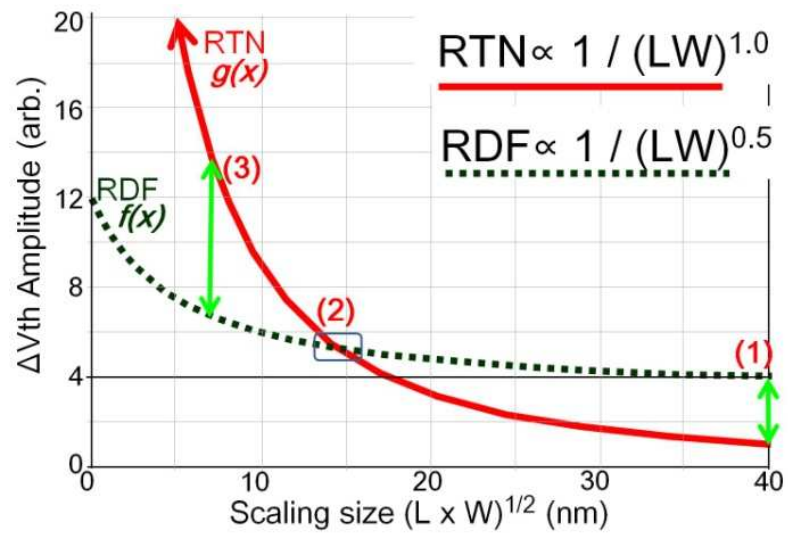

(a)

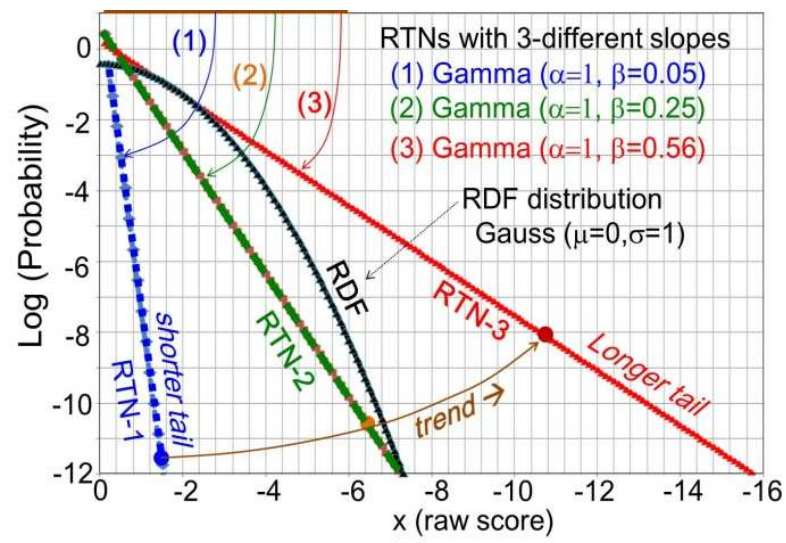

(b)

Fig. 2. (a) Increasing pace comparisons of RDF and RTN, (b) Relationship of tail length of RDF and 3-type of RTN, RTN-1, RTN-2, and RTN-3, where RTN-n corresponds to RTN at the point of (n), n=1,2,3 in Fig. 2(a).
MOSFET channel length and width [3, 4], respectively. $\Delta \mathrm{V}$ th is equivalent to $1.414 \times \sigma \mathrm{V}$ th.

Assuming that LW is scaled down to a half size $(0.5 \mathrm{x})$ for every process generation, the $\sigma \mathrm{V}$ th increasing paces of the RTN becomes $1.4 \mathrm{x}$ faster than that of the RDF. In addition, because the advanced CMOS device tends to change to much less-dopant body devices, such as FinFET, ultra-thin body SOI and nanowire FET, the RDF increasing the speed can be reduced, resulting in an increasing pace $[3,4]$.

As a result, the amplitude for the RTN $(g(x))$ will at some time exceed the amplitude for the $\operatorname{RDF}(f(x))$, as shown in Figs. 2(a) and (b). According to [3, 4], it might occur around the $15 \mathrm{~nm}$ scaled CMOS era, which is expected in the near future. A change in the relationship between $\boldsymbol{g}(\boldsymbol{x})$ and $\boldsymbol{f}(\boldsymbol{x})$ can be observed when comparing the relationship in Fig. 2(a) with that in Fig. 2(b).

The remainder of this paper is organized as follows. Section II discusses the issues on the deconvolution. Section III examines the deconvolution error comparisons and propose the new algorithm to address the issues. The extraction results of the proposed one are also discussed in this section followed by the conclusion in section IV.

\section{CONVOLUTION/DECONVOLUTION OF RTN AND RDF}

\section{Concept of Convolution and Deconvolution}

First of all in this section, the relationship between the convolution $(\otimes)$ and deconvolution $\left(\otimes^{-1}\right)$ is explained, where $\otimes$ and $\otimes^{-1}$ being used in this paper are arithmetic symbols of operation of the convolution and deconvolution, respectively.

Fig. 3(a) shows the concept for the required convolution methods to extrapolate the entire MV distribution based on the given data of the RDF and the RTN. Fig. 3(b) recounts the following scenarios: the target specs were predefined in advance. The RTN distribution was also predefined or given. Therefore, the required truncating point (TP), corresponding to a certain minimum operating voltage $\left(V_{D D, \min }\right)$, which is used for the screening test $V_{D D}$, can be determined. The TP should be decided based on the RDF distributions to avoid any out of specs after the shipment. Fig. 3(c) assumes the different scenarios: both of the target 
(a)

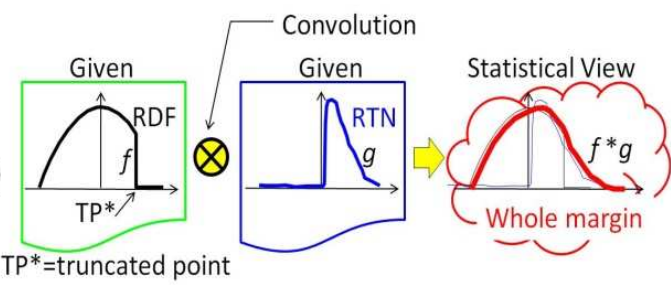

(b)

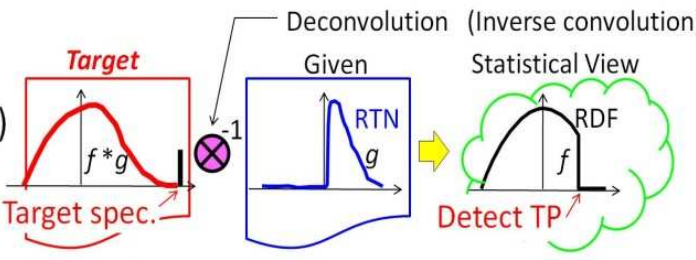

(c)

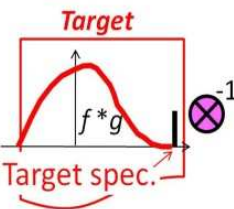

Given

(d)

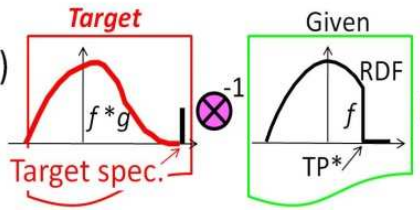

Statistical View

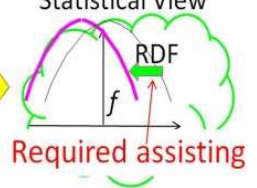

Statistical View

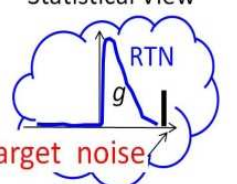

Fig. 3. Comparisons of the convolution and deconvolution (a) convolution of the given data of RDF and RTN, (b)-(d) examples of deconvolution (inverse problem) required to detect the unknown factors based on the pre-defined target specifications: (b) detecting TP, (c) required assisting amount, (d) noise level needed to keep the MV tail within the target spec.

specs and the RTN distribution are predefined or given. Therefore, how much the overall MV can be shifted using the MV assisted circuit schemes (ASSTS) can be determined $[8,9]$.

This can be used to set the target of the SRAM circuit designers. Fig. 3(d) shows another scenario. The target specs and the truncated RDF distribution are predefined or given. Therefore, the device target for the reduced level of the RTN amplitude can be specified. This can be used to set the target of process device engineers.

\section{Computing of Convolution and Deconvolution}

Table 1(a) presents the convolution given by the equation. Since the convolution of $\boldsymbol{f}$ with $\boldsymbol{g}$ can be considered as the forward problem, if the mathematical equation and the parameters at the segmentation of $\boldsymbol{f}_{\boldsymbol{l}}-\boldsymbol{f}_{\boldsymbol{n}}$ and $\boldsymbol{g}_{\boldsymbol{l}} \boldsymbol{g}_{\boldsymbol{n}}$, are given, the observation of $\boldsymbol{h}_{\boldsymbol{l}}-\boldsymbol{h}_{\boldsymbol{n}}$ can be calculated easily. This operation plays the role of low-

Table 1. Comparisons of computing steps of convolution and deconvolution.

(a) Convolution $\mathrm{h}=\mathrm{f} \otimes \mathrm{g}$

\begin{tabular}{|l|}
\hline $\mathrm{h}_{1}<=\mathrm{f}_{1} * \mathrm{~g}_{1}$ \\
\hline $\mathrm{h}_{2}<=\mathrm{f}_{1} * \mathrm{~g}_{2}+\mathrm{f}_{2} * \mathrm{~g}_{1}$ \\
$\mathrm{~h}_{3}<=\mathrm{f}_{1} * \mathrm{~g}_{3}+\mathrm{f}_{2} \mathrm{~g}_{2}+\mathrm{f}_{3} * \mathrm{~g}_{1}$ \\
\hline$\vdots$ \\
\hline \\
$\mathrm{h}_{\mathrm{n}}<=\mathrm{f}_{1}{ } \mathrm{~g}_{\mathrm{n}}+\mathrm{f}_{2} * \mathrm{~g}_{\mathrm{n}-1}+\mathrm{f}_{3} * \mathrm{~g}_{\mathrm{n}-2}+\mathrm{f}_{4} * \mathrm{~g}_{\mathrm{n}-3} \ldots \ldots . .+\mathrm{f}_{\mathrm{n}} * \mathrm{~g}_{1}$ \\
\hline
\end{tabular}

(b) Deconvolution $\mathrm{g}=\mathrm{h} \otimes^{-1} \mathrm{f}$

\begin{tabular}{|l|}
\hline $\mathrm{g}_{1}<=\mathrm{h}_{1} / \mathrm{f}_{1}$ \\
$\mathrm{~g}_{2}<=\left(\mathrm{h}_{2}-\mathrm{f}_{2} * \mathrm{~g}_{1}\right) / \mathrm{f}_{1}$ \\
$\mathrm{~g}_{3}<=\left(\mathrm{h}_{3}-\mathrm{f}_{2} * \mathrm{~g}_{2}-\mathrm{f}_{3} * \mathrm{~g}_{1}\right) / \mathrm{f}_{1}$ \\
\hline$\vdots$ \\
\hline \\
\hline $\mathrm{g}_{\mathrm{n}}<=\left(\mathrm{h}_{\mathrm{n}}-\mathrm{f}_{2} * \mathrm{~g}_{\mathrm{n}-1}-\mathrm{f}_{3} * \mathrm{~g}_{\mathrm{n}-2}-\mathrm{f}_{4} * \mathrm{~g}_{\mathrm{n}-3} \quad \ldots \ldots \ldots-\mathrm{f}_{\mathrm{n}} * \mathrm{~g}_{1}\right) / \mathrm{f}_{1}$ \\
\hline
\end{tabular}

pass filtering, resulting in $\boldsymbol{h}=\boldsymbol{f} \otimes \boldsymbol{g}$ being a smoothed curve.

The deconvolution can be calculated based on the equation in Table 1(b). If the observation of $\boldsymbol{h}_{\boldsymbol{T}}-\boldsymbol{h}_{\boldsymbol{n}}$ and the parameter of $f_{l}-f_{n}$ at the segmentation are given with the mathematical equation, the unknown parameter of $\boldsymbol{g}_{\boldsymbol{I}^{-}}$ $\boldsymbol{g}_{n}$ can be calculated easily under the limited conditions.

On the other hand, because the deconvolution needs to be considered as an inverse problem, the invert operation, i.e., high-pass filtering, needs to be performed, as shown in Table 1(b).

\section{Issues Facing Deconvolution Operations}

The deconvolution can be calculated based on the equation in Table 1(b). If the observation of $\boldsymbol{h}_{\boldsymbol{l}}-\boldsymbol{h}_{\boldsymbol{n}}$ and the parameter of $f_{i}-f_{n}$ at the segmentation are given with the mathematical equation, the unknown parameter of $\boldsymbol{g}_{\boldsymbol{I}^{-}}$ $\boldsymbol{g}_{\boldsymbol{n}}$ can be calculated easily under the limited conditions.

On the other hand, because the deconvolution needs to be considered as an inverse problem, the invert operation, i.e., high-pass filtering, needs to be performed, as shown in Table 1(b). This causes the issues facing the inverse operations: (1) division by zero and Gibbs phenomenon, resulting in a ringing curve. As shown in Fig. 4, the deconvolution of the RTN causes abnormal folding and ringing, resulting in significant deviation of the RTN distribution from the expected curve. This stems from the 

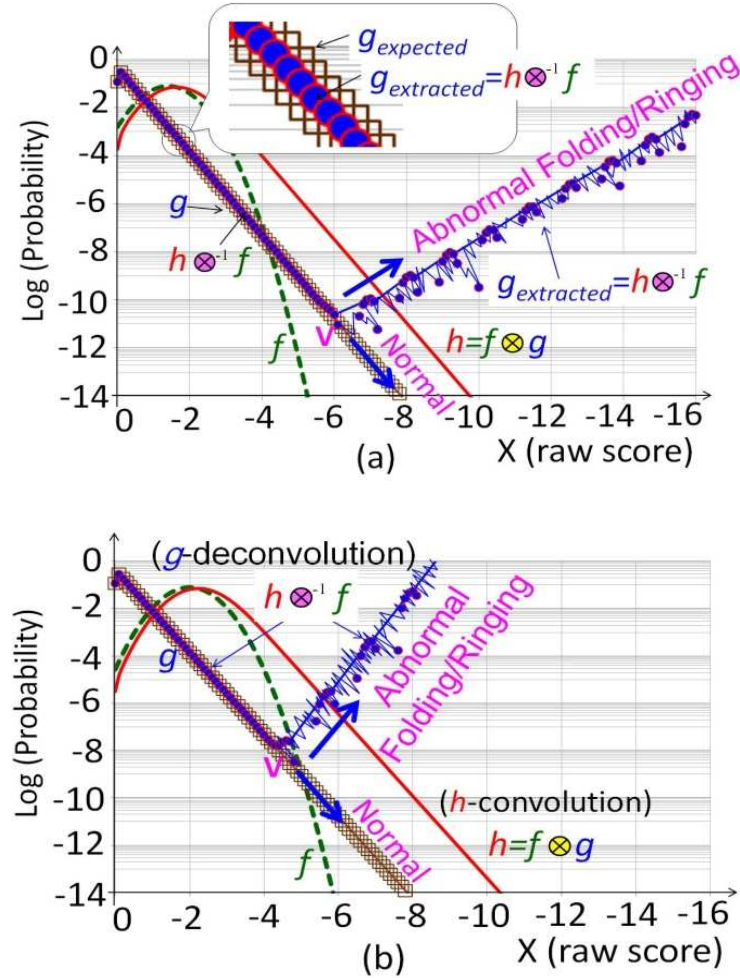

Fig. 4. Abnormal phenomenon of V-shaped folding and ringing when using the conventional deconvolution algorithm. The difference between (a) and (b) is just the x-phase shift of $f$.

required invert operation, i.e. high-pass filtering to perform the deconvolution. The point of "V" where the error exceeds a certain level, resulting in occurrence of "V-shaped" abnormal error is quite sensitive to the relationship of the gradients among $\boldsymbol{f}, \boldsymbol{g}$ and $\boldsymbol{h}$, as can be seen when comparing those of Figs. 4(a) and (b).

It is found that the ringing and folding $\mathrm{V}$-shaped error also happen in the case of assuming the relationship of the RDF $\boldsymbol{f}(\boldsymbol{x})$ and RTN-2 $\boldsymbol{g}(\boldsymbol{x})$ (see Fig. 2(b)). This error makes the deconvoluted RTN distribution $\boldsymbol{g}(\boldsymbol{x})$ significantly deviated from the expected curve, as shown in Fig. 5(a). This stems from the required invert operation, i.e., differentiation and feedbacks, resulting in accumulating of the errors, as shown in Fig. 5(b). Once the accumulated error level exceeds a certain level, the ringing are excited and the error is amplified, as shown in Fig. 5(a). This sort of high-pass filtering behavior makes the output more sensitive to the noise and the error, resulting in an unstable operation $[7,8]$.

The purpose of this work is to propose the three novel deconvolution methods for the SRAM-designs, which enable: (1) to substantially circumvent the abnormal V-

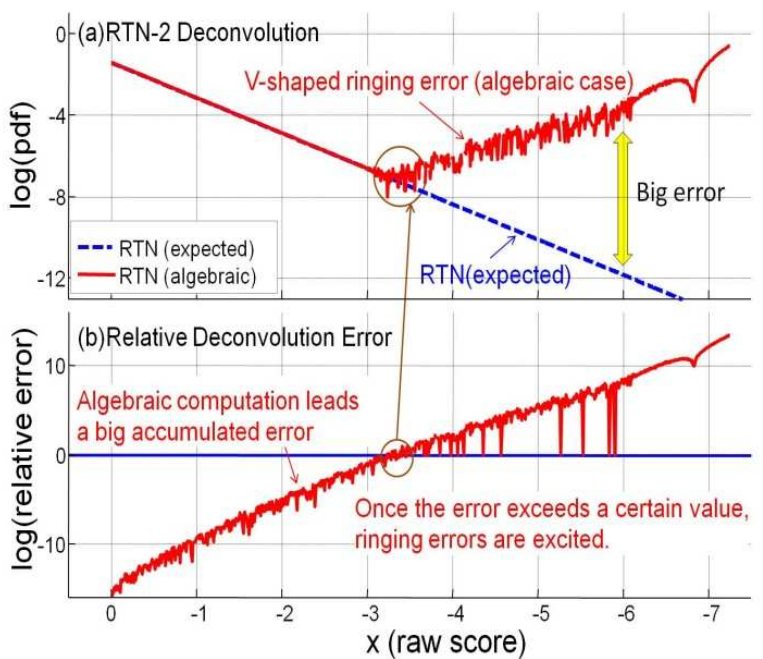

Fig. 5. Issue of V-shaped error in deconvoluion process using algebraic way where division by zero can happen.

shaped ringing errors by eliminating the need of the inverse operation, (2) to increase the deconvolution accuracy in the tail region where its pdf is less than $10^{-12}$. Since the SRAM fail probability is extremely rare-event level (pdf is less than $10^{-12}$ ), the degree of precision for the tail distribution gives a big impact on the accuracy of the fail-bit count (FBC) prediction. Unlike the conventional optimization problem, which tends to neglect the rare-event probability zone, the proposed idea tries to keep a sharp eye on the rare event probability area by introducing the segmented optimization and (3) to guarantee the good enough deconvolution precision even if the RTN distribution comprises the complex gamma mixtures with the multiple convex and concave folding points.

To the best of our knowledge, our work [9] was the first time to present the deconvolution algorithm for SRAM-designs featuring an iterative and adaptively segmented forward-problem based deconvolution (ASDCN) process and enabling to achieve the above mentioned three objects.

\section{Proposed Adaptively Segmented F ORWARD-PROBLEM BASED I TERATIVE DeCONVOLUTION Algorithm}

\section{Forward-Problem Based Algorithm}

The key feature of the forward based deconvolution 


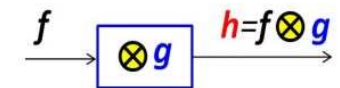

Forward problem (Convolution) (a) Convolution

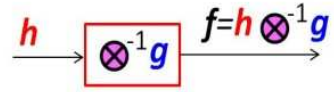

Inverse problem (Deconvolution) (b) Algebraic way ( division by zero)

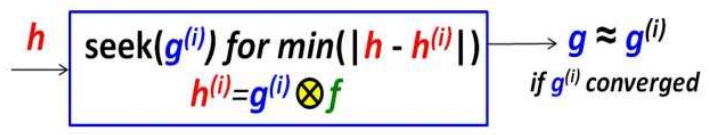

(c) Forward based deconvolution w/o segmentation

Fig. 6. Deconvolution algorithm comparisons between (b) the conventional algebraic, (c) proposed one that uses only (a) convolution instead of (b) algebraic deconvolution process.

algorithm is not to use the inverse operation (see Fig. 6(b)) such as differentiation and division by zero at all, as shown in Fig. 6(c). Instead, we solve the optimization problem that seeks $\boldsymbol{g}^{(i)}$ for minimizing $\left(\left|\boldsymbol{h}-\boldsymbol{h}^{(i)}\right|\right)$, where $\boldsymbol{h}^{(i)}$ is the convolution of $\boldsymbol{g}^{(i)}$ with $\boldsymbol{f}$, as shown in Fig. 6(c). We use "fminsearch" in MATLAB ${ }^{\circledR}$ to seek the minimum of unconstrained multivariable function, which allows a derivative-free method. The distribution of $\boldsymbol{g}^{(i)}$ is approximated by gamma distribution with three parameters of $\alpha$ (shape), $\beta$ (inverse scale) and $\kappa$ (peak value). In the proposed algorithm, $\boldsymbol{h}^{(i)}$ is defined as the convolution of $\boldsymbol{h}^{(i)}=\boldsymbol{g}^{(i)} \otimes \boldsymbol{f}$ (see Fig. 6(c)). Thanks to avoiding the inverse operation, the behavior of the deconvolution process becomes insensitive to the noise and the error like the low-pass filtering. As a result, the accumulated deconvolution errors are significantly suppressed and its error level is reduced by $>10^{17}$ at raw score $x=-6$, as shown in Fig. 7. Any ringing noises and $\mathrm{V}$-shaped errors are not observed any more when using the forward problem based deconvolution algorithm.

\section{Limitation of Forward-Problem Based Algorithm}

As proposed in Fig. 6(c), the forward problem-based deconvolution algorithm takes some optimization process to find the best $\boldsymbol{g}^{(i)}$ that minimize the $\left|\boldsymbol{h}-\boldsymbol{h}^{(i)}\right|$. In this application, however, since the distribution type is sort of exponential, the difference in the pdf level between $\mathrm{x}=0$ and $\mathrm{x}=-6$ is very huge, e.g., 4 orders of magnitude, as can be seen in Fig. 8(b). The conventional optimization problem prioritizes the higher frequent probability around $\mathrm{x}=0$ because the $\mathrm{pdf}$ around there dominates the overall cdf. As a result, unfortunately, the cdf error is best reduced around $\mathrm{x}=0$, while leaving the errors in the

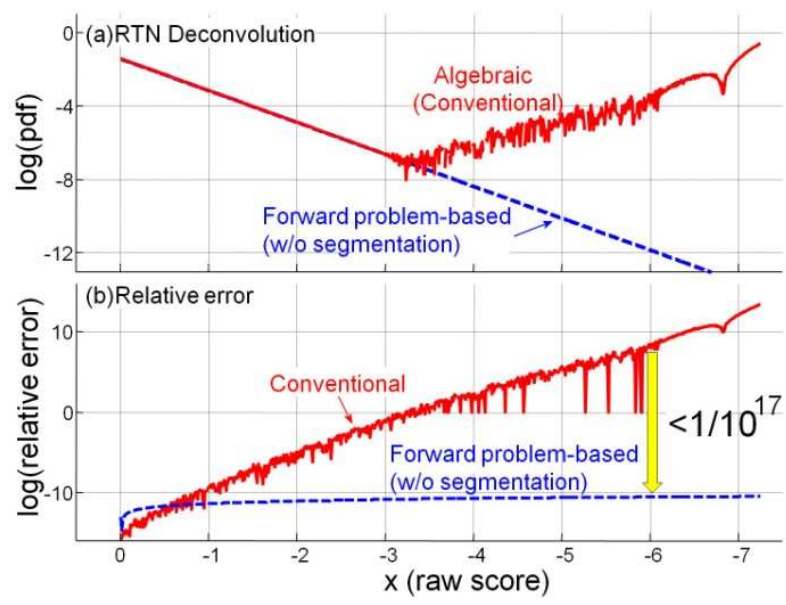

Fig. 7. Comparison of RTN deconvolution results between the algebraic and forward problem-based algorithms.

(a) Entire Area-at-Once-Optimization (w/o segmentation)

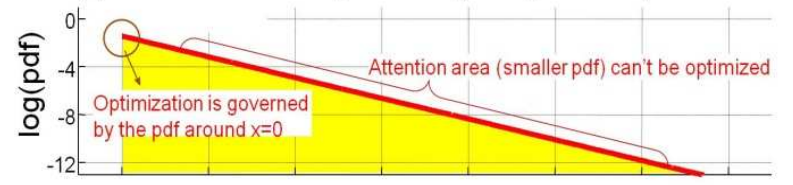

(b)Relative deconvolution error

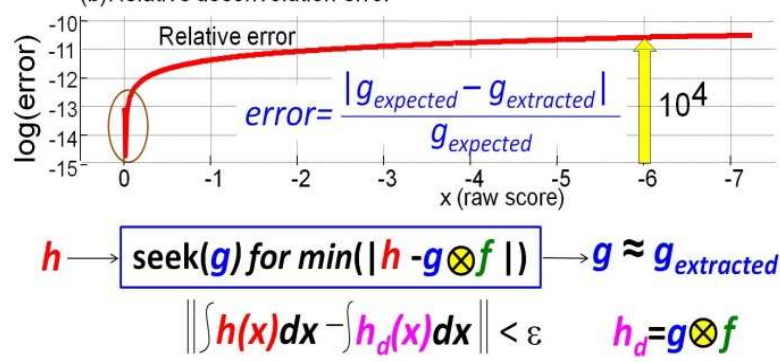

Fig. 8. Limitation of forward-problem based algorithm.

tail region $(\mathrm{x}=-6)$ as it is.

When strictly comparing the relative errors across all $\mathrm{x}$-regions, it has an x-position dependency and becomes larger as the raw score $\mathrm{x}$ is increased, as shown in Fig. 8(b). When considering an actual application for the failbit count (FBC) prediction, the degree of precision for the tail distribution gives a huge impact on the accuracy of the FBC prediction because the SRAM fail probability is extremely rare-event level ( $\mathrm{pdf}$ is less than $10^{-12}$ ).

\section{Proposed Segmented Deconvolution Algorithm}

To address this issue, we propose a novel algorithm featuring an iterative and segmented forward problem based deconvolution (SDCN). The key concept is shown in Fig. 9. $\boldsymbol{h}^{(i)}$ is defined as the convolution of the 

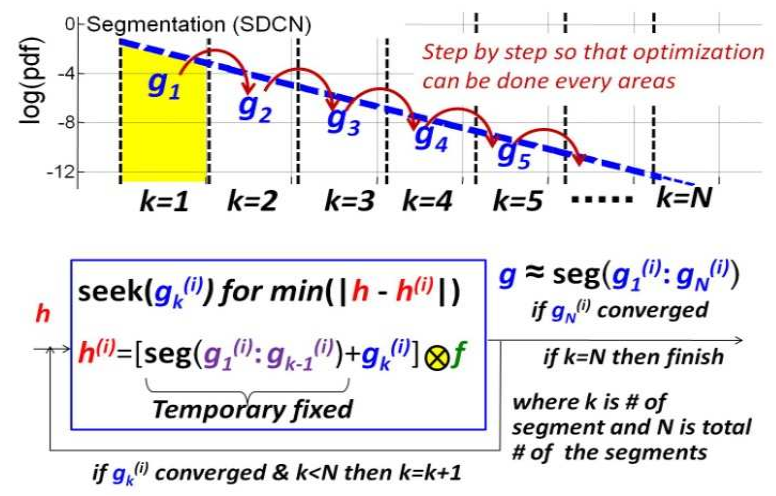

Fig. 9. Segmented deconvolution algorithm.

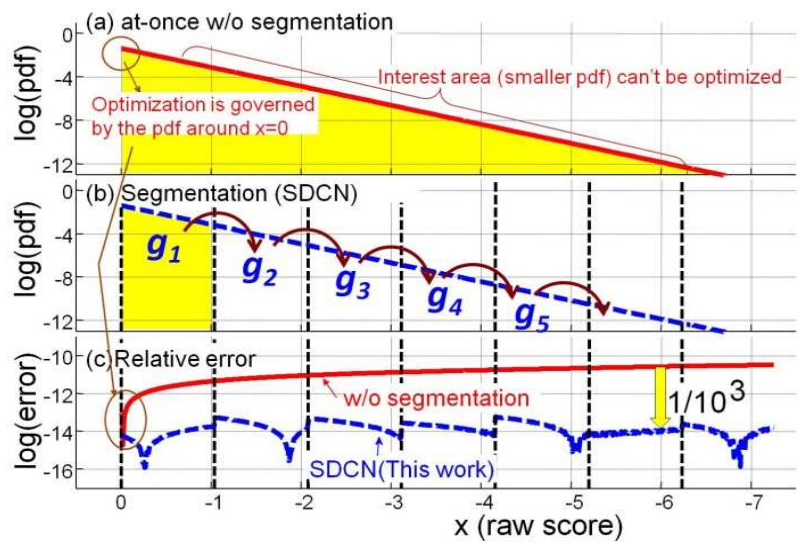

Fig. 10. Segmented deconvolution algorithm.

summation of the line-segment of $\left(\boldsymbol{g}_{\boldsymbol{l}}^{(i)}: \boldsymbol{g}_{\boldsymbol{k}-\boldsymbol{l}}{ }^{(i)}\right)$ and $\boldsymbol{g}_{\boldsymbol{k}}^{(i)}$ with $\boldsymbol{f}$, i.e., $\boldsymbol{h}^{(i)}=\left[\operatorname{seg}\left(\boldsymbol{g}_{\boldsymbol{l}}{ }^{(i)}: \boldsymbol{g}_{\boldsymbol{k}-\boldsymbol{I}}{ }^{(i)}\right)+\boldsymbol{g}_{\boldsymbol{k}}{ }^{(i)}\right] \otimes \boldsymbol{f}$ (see Fig. 9)

The process of seeking the best $\boldsymbol{g}^{(i)}$ follows the sequentially step by step manner, i.e., starting from around $\mathrm{x}=0$ and finally focusing on the important zone of the rare-event so that each optimization step cannot be interfered with by the other higher sensitivity zone. Once found the best $\boldsymbol{g}^{(i)}$ in each segmentation, its value is temporally fixed until the next iteration process.

Thanks to this algorithm, the error is reduced by $10^{3}$ compared with the non-segmentation case, as shown in Fig. 10. It can be seen in Fig. 10 that the each optimization in individual segmentation is successfully done, resulting in no dependency of the position of the segmentation.

\section{RTN Tail Length Dependency}

In this session, the RTN tail length (tail slope) dependencies on the error are compared. As can be seen

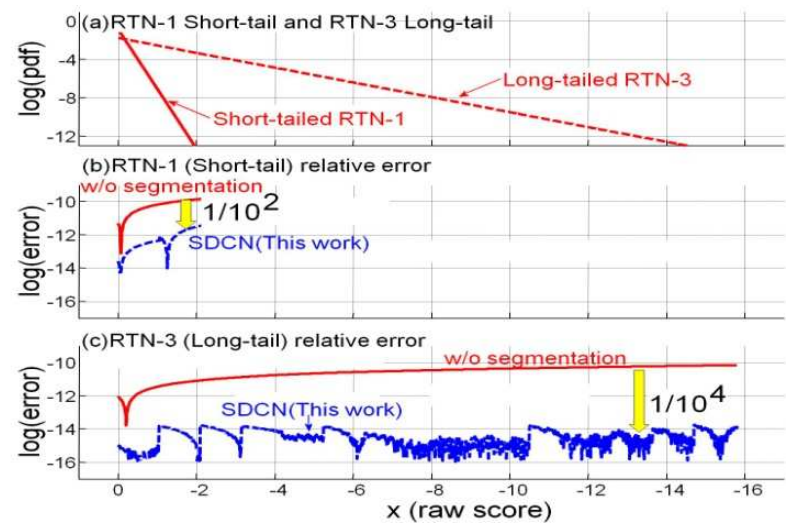

Fig. 11. Comparisons of the effectiveness of the proposed segmented algorithm between the short (RTN-1) and long tailed RTN (RTN-3).
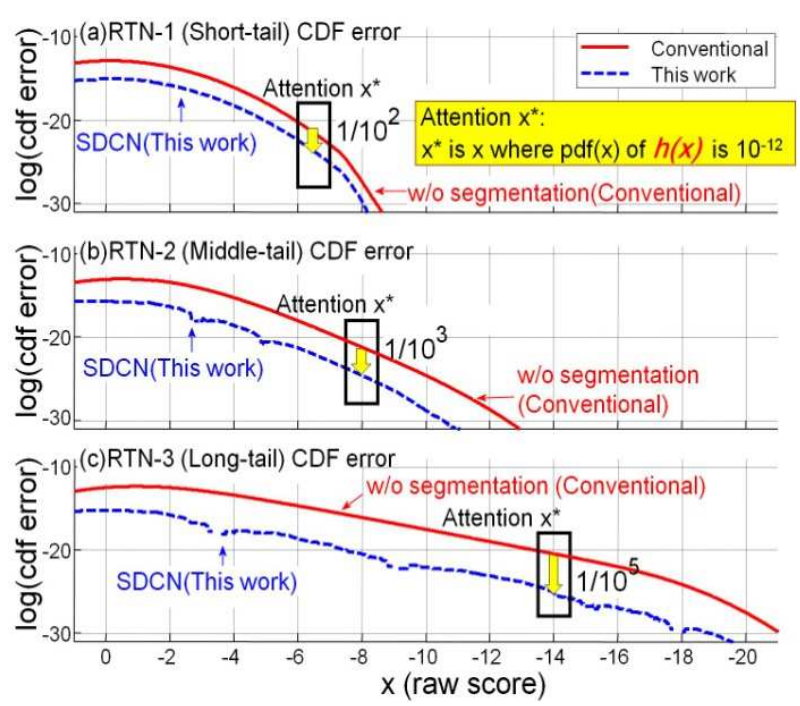

Fig. 12. Comparisons of cdf error (i.e., fail bit count error) between the cases w/ and w/o the proposed SDCN algorithm for different RTN tail length.

in Figs. 11(b) and (c), it is found that the proposed SDCN can reduce the error for the short tailed and long tailed RTN by $10^{2}$ and $10^{4}$, respectively, compared with the case without any segmented optimizations.

Since the final target of this work is to increase the accuracy of the FBC prediction, the precision of the cumulative distribution function (cdf) of $\boldsymbol{h}(\boldsymbol{x})$ is the key to the success of this work. Fig. 12 shows the comparisons of the cdf errors for the several cases with a different RTN tail length. The box represents for the attention x-zone where its pdf level of $\boldsymbol{h}(\boldsymbol{x})$ is about $10^{-12}$, i.e., rare event level. This zone is referred to as "attention $\mathrm{x}$ ". When compared with the cdf error at this x point, it is 


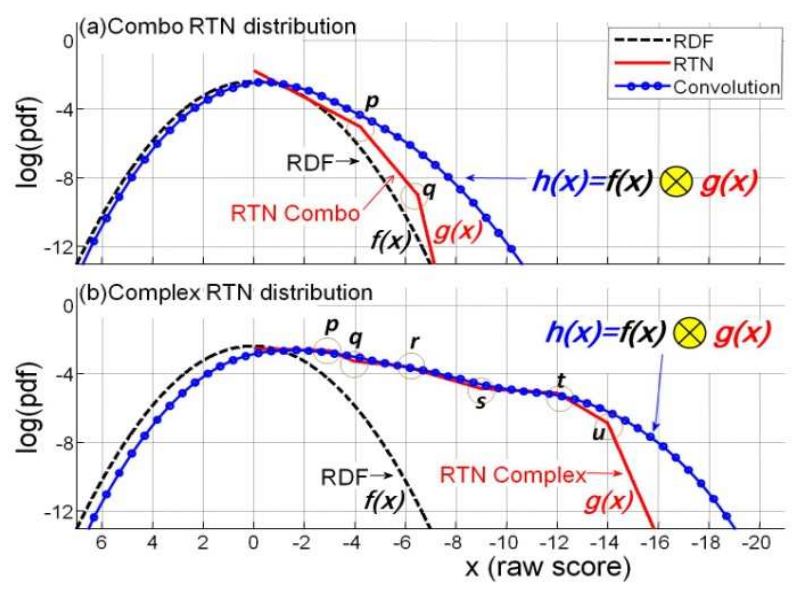

Fig. 13. 2-types of RTN with (a) multiple convex folding points, (b) mixtures of the convex and concave folding points.

found that the proposed SDCN can increase the accuracy of the FBC prediction by $10^{2}, 10^{3}$, and $10^{5}$, respectively.

\section{Limitation of SDCN Algrithm}

In session IV, we assume that the RTN distribution obeys a single gamma distribution with different tail slope and length.

However, we may need to assume RTN distribution with more complex gamma mixtures with various sloped segments, as shown in Fig. 13. In this paper, we refer to the RTN distribution with the multiple convex folding points $(p, q)$ and the mixtures of the convex and concave folding points (p,q,r,s,t,u), i.e., (a) "RTN combo" and (b) "RTN complex", respectively, as shown in Fig. 13.

As can be seen in Fig. 13, the distribution shape gives a big impact on the distribution of the convolution of RDF with RTN. In that sense, we have to guarantee the precision level of the RTN deconvolution whatever its slope and length of the RTN tail is.

To make more clear the effectiveness and remaining the issues of the proposed algorithm SDCN, the demonstration results for the case of combo is shown in Fig. 14(b).

Fig. 14(a) shows the deconvolution result for the combo RTN with two convex folding points $(p, q)$ by using the SDCN algorithm. As can be seen in Fig. 14(b), the errors in the segment that includes the folding points $(p, q)$ are 15-orders of magnitude larger than those in other segments. This is the remaining issue confronting the proposed SDCN when adapting to the combo and
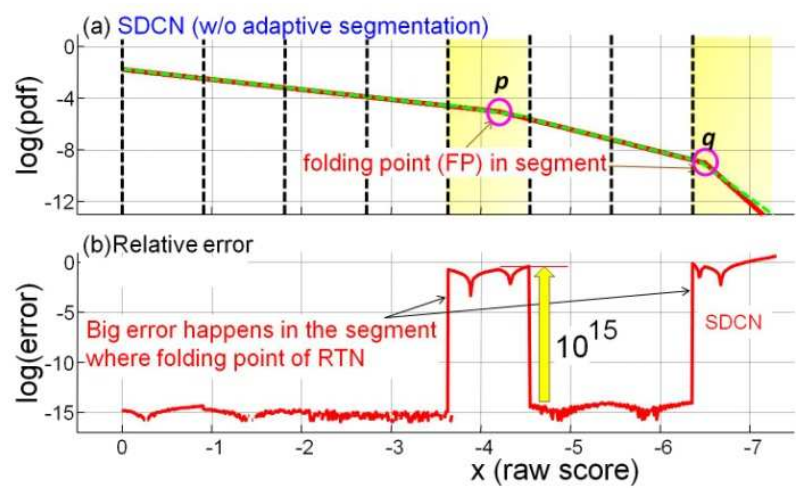

Fig. 14. Limitation of SDCN algorithm when there is the folding point in the segmentation such as $\boldsymbol{p}$ and $\boldsymbol{q}$.
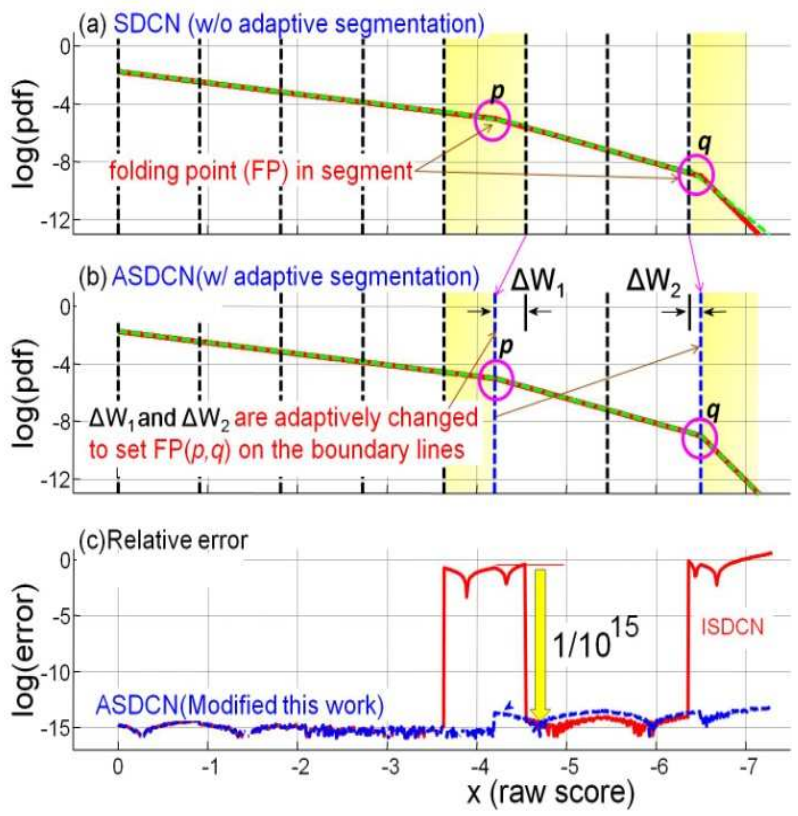

Fig. 15. Effects of the proposed ASDCN algorithm on the error reduction for the combo-RTN distribution.

complex typed RTN tail distributions.

\section{Proposed Adaplitively Segmentation Algorithm}

To address this issue, we propose that the segmentation width is adaptively changed by $\Delta \mathrm{W}_{1}$ and $\Delta \mathrm{W}_{2}$ such that the error can be minimized (i.e, $\min \mid \boldsymbol{h}$ $\boldsymbol{h}^{\prime} \mid$, where $\boldsymbol{h}^{\prime}=\boldsymbol{f} \otimes \boldsymbol{g}^{\prime}$ and $\left.\boldsymbol{g} \boldsymbol{\prime}^{\prime} \boldsymbol{h} \otimes{ }^{-1} \boldsymbol{f}\right)$. The $\Delta \mathrm{W}_{\mathrm{n}}$ is the shift amount required to set the boundary line of the segmentations always just on folding point (FP), as shown in Fig. 15(b).

Thanks to the proposed segmentations, the error is reduced to $1 / 10^{15}$ that of the original result, as shown in 

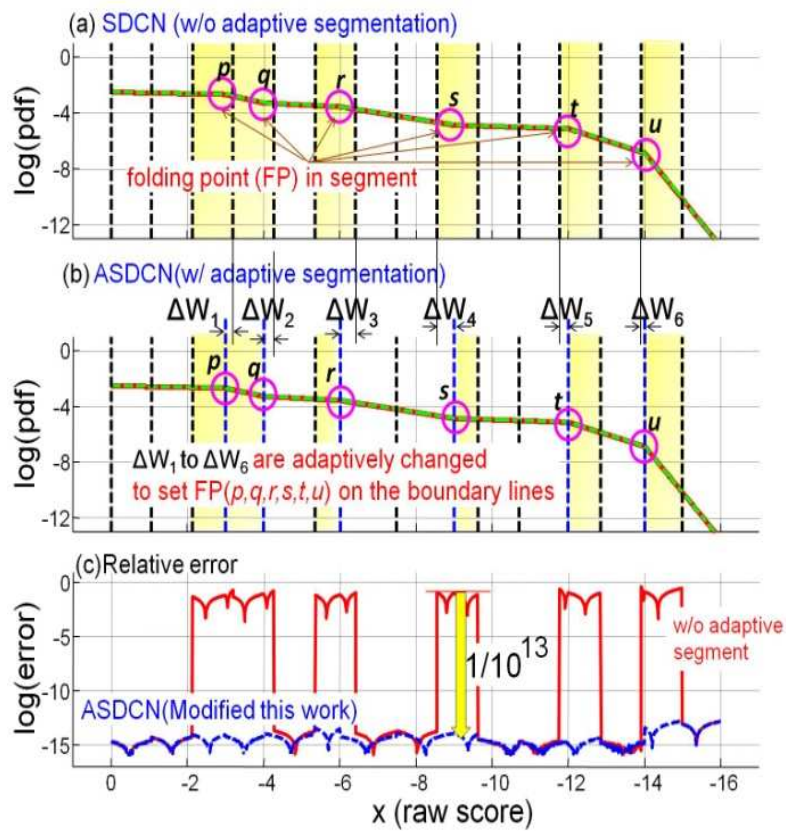

Fig. 16. Effects of the proposed ASDCN algorithm on the error reduction for the complex-RTN distribution.

Fig. 15(c). We refer to this modified work as "adaptively segmented forward-problem based iterative deconvolution" (ASDCN).

Figs. 16(a) and (b) show the comparison of the deconvolution results for the complex RTN with 4convex $(p, r, t, u)$ and 2 -concave $(q, s)$ folding points between the two cases of using the original SDCN and the modified algorithm (ASDCN), respectively. The $\Delta \mathrm{W}_{\mathrm{n}}(\mathrm{n}=1$ to 6$)$ are the shift amounts required to set the boundary line of the segmentations just on the folding point (FP), as shown in Fig. 16(b). It is found that the error for ASDCN is reduced to $1 / 10^{13}$ that of the SDCN, as shown in Fig. 16(c).

Fig. 17 shows the comparisons of the cdf error (i.e., FBC prediction error) between the combo and complex RTN with the different RTN tail shape and length. When compared with cdf error at the $\mathrm{x}$ point in the interest area, it is found that the modified proposed work (ASDCN) can increase the accuracy of the FBC prediction by $10^{10}$ fold compared with the conventional case that doesn't use the adaptive segmentation.

\section{Discussions AND CONCLUSIONS}

We have proposed the deconvolution techniques (SDCN and ASDCN) enabling to successfully

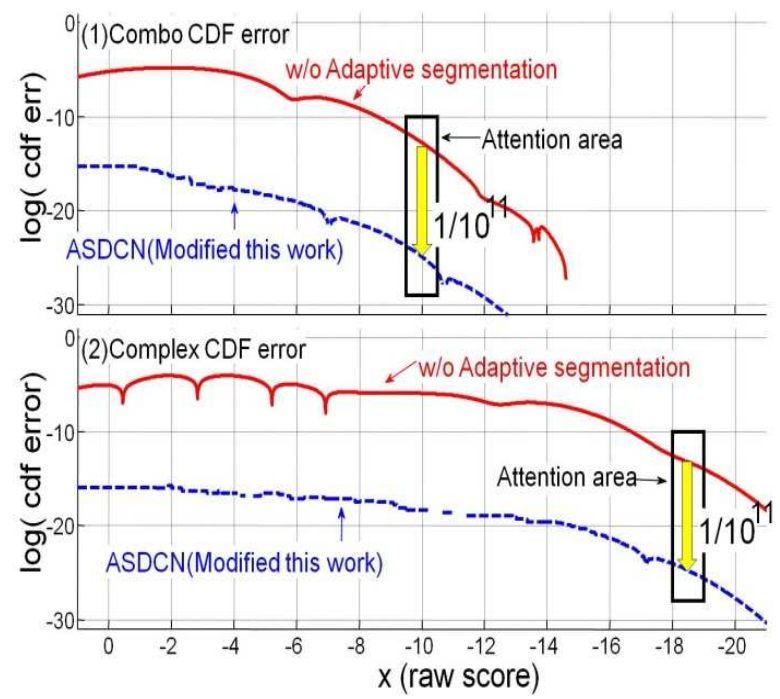

Fig. 17. Effects of the proposed ASDCN algorithm on the cdferror reduction for the combo and complex-RTN distribution. This work reduces the FBC errors by $10^{10}$.

Table 2. Summary and Comparisons of RTN $g(x)$ deconvolution errors and its impact on cdf errors of $h(x)$.

\begin{tabular}{|c|c|c|c|c|}
\hline & & RTN1 & RTN2 & RTN3 \\
\hline \multirow{2}{*}{ ERR-A } & $\begin{array}{c}\text { Conventional } \\
\text { (w/o segmentation) }\end{array}$ & $1 e-10$ & $5 e-11$ & $9 e-11$ \\
\hline & SDCN & $2 \mathrm{e}-12$ & $1 e-14$ & $9 e-15$ \\
\hline \multirow{3}{*}{ ERR-C } & $\begin{array}{c}\text { Conventional } \\
\text { (w/o segmentation) }\end{array}$ & $1 e-21$ & $1 e-21$ & $1 \mathrm{e}-20$ \\
\hline & SDCN & $1 e-23$ & $1 e-24$ & $1 e-25$ \\
\hline & & \multicolumn{2}{|l|}{ Combo } & Complex \\
\hline \multirow{2}{*}{ ERR-B } & SDCN & \multicolumn{2}{|l|}{$5 e-1$} & $1 e-1$ \\
\hline & ASDCN & \multicolumn{2}{|l|}{$1 e-15$} & $1 e-14$ \\
\hline \multirow{2}{*}{ ERR-C } & SDCN & \multicolumn{2}{|l|}{$1 e-13$} & $1 e-13$ \\
\hline & ASDCN & \multicolumn{2}{|l|}{$1 e-24$} & $1 e-24$ \\
\hline
\end{tabular}

ERR-A : RTN relative error in segment without folding point ERR-B : RTN relative error in segment with folding point ERR-C : Cdf relative error for $\operatorname{cdf}(X a)$ of $h(x)$ where $X a$ is for $\operatorname{pdf}(X a)=10^{-12}$

circumvent the issue on an abnormal V-shaped error confronting the conventional algebraic based-inverse computation process. The effectiveness of the proposed techniques have been demonstrated for the first time with applying to a real analysis for the effects of the RTN and the RDF on the overall SRAM margin variations.

Table 2 shows that the SDCN reduces its relative deconvolution errors for RTN-1, RTN-2, and RTN-3 by about $10^{2}, 10^{3}$ and $10^{4}$ fold, respectively. As a result, the fail-bit-count prediction errors for them are reduced by about $10^{2}, 10^{3}$ and $10^{5}$ fold, respectively thanks to the suppressed deconvolution errors.

The proposed ASDCN solves the issues confronting 
the SDCN technique when applying for RTN distributions with folding points (Combo and Complex). It is found that $\mathrm{ASDCN}$ reduces its relative deconvolution errors for Combo and Complex by about $10^{14}$ and $10^{13}$ fold, respectively. As a result, the fail-bitcount prediction errors for them are reduced by about $10^{11}$ thanks to the suppressed deconvolution errors.

\section{ACKNOWLEDGMENTS}

This research was supported by MEXT/JSPS KAKENHI Grant Number of 23560424 and grant from Information Science Laboratory of Fukuoka Institute of Technology.

\section{REFERENCES}

[1] K. Takeuchi, et al, "Comprehensive SRAM Design Methodology for RTN Reliability”, Digest of IEEE Symposium on VLSI Technology, (2011), pp. 130131

[2] K. Takeuchi, et al, "Direct Observation of RTNinduced SRAM Failure by Accelerated Testing and Its Application to Product Relaiability Assesment", Digest of IEEE Symp on VLSI Tech, (2010), pp. 189-190

[3] M. Yamaoka, et al, "Evaluation Methodology for Random Telegraph Noise Effects in SRAM Arrays", Digest of IEEE IEDM, (2011), pp. 745748

[4] X. Wang, et al, "RTS amplitude distribution in 20nm SOI FinFETs subject to Statistical Variability”, SISPAD 2012, (2012) pp.296-299.

[5] X. Wang, et al, "Simulation Study of Dominant Statistical Variability Sources in 32-nm Highk/Metal Gate CMOS", IEEE Electron Device Letters - , vol. 33, no. 5, (2012), pp. 643-645, 2012.

[6] Q. Shi, et al, "Application of Iterative Deconvolution for Wire Fault Location via Reflectometry", Digest of IEEEInternational Symposium on Instrumentation \& Measurement, Sensor Network and Automation (IMSNA), (2012), pp. 102-106

[7] W. Somha and H. Yamauchi, "Convolution/ Deconvolution SRAM Analyses for Complex Gamma Mixtures RTN Distributions", Digest of
ICICDT-2013,(2013), pp. 33-36,

[8] W. Somha and H.Yamauchi, "A V-shaped Deconvolution Error Suppression Technique for SRAM Analyses of Long-Tail Gamma Mixtures Random Telegraph Noise Distribution Effects", Digestof ITC-CSCC-2013,(2013) pp. 297-299

[9] W.Somha, H.Yamauchi, Ma yuyu, "Iterative and Adaptively Segmented Forward Problem Based Non-Blind Deconvolution Technique for Analyzing SRAM Margin Variation Effects",International Soc Design Conference (ISOCC2013), November, 2013, pp.184-187

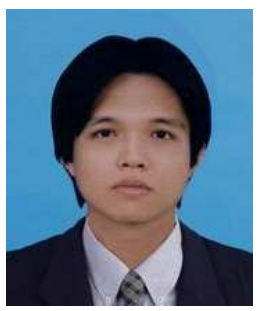

Worawit Somha received his master degree in electrical engineering from King Mongkut's Institute of Technology Ladkrabang (KMITL), Bangkok, Thailand. His master thesis was on "Vector Quantizers for Speech Coding and there Implementation on TMS-320C30". Since 1995 he has been a lecturer for bachelor degree students in subject of "Introduction to Digital Signal Processing" at KMITL as an assistance professor, and his research area is speech coding. Since 2012 he won the scholarship from KMITL for the Dept. of Eng. student program and now being pursuing a $\mathrm{PhD}$ degree with a major in intelligence information system engineering at Fukuoka Institute of Technology. He is a member of the IEICE since 2012.Mr. Somha received the IEEK ISOCC2013 Best Paper Award. 


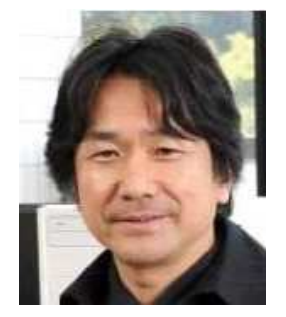

Hiroyuki Yamauchi received his Ph.D. degree in engineering from Kyushu University, Fukuoka, Japan, in 1997. His doctoral dissertation was on "Low Power Technologies for BatteryOperated Semiconductor Random Access Memories". In 1985 he joined the Semiconductor Research Center, Panasonic, Osaka, Japan. From 1985 to 1987 he worked on the research of the submicron MOS FET model-parameter extraction for circuit simulation and examined the sensitivity of the scaled sense amplifier for ultrahigh-density DRAM's which was presented at the 1989 Symposium on VLSI Circuits. From 1988 to 1994, he was engaged in the research and development of 16-Mb CMOS DRAM's including the battery-operated high-speed $16 \mathrm{Mbit}$ CMOS DRAM and the ultra low-power, three times longer, self-refresh DRAM which were presented at the 1993 and 1995 ISSCC, respectively. He also presented the charge-recycling bus architecture and low-voltage operated high-speed VLSI's, including $0.5 \mathrm{~V} / 100 \mathrm{MHz}-$ operated SRAM and Gate-Over-Driving CMOS architecture, which were presented at the Symposium on VLSI Circuits in 1994 and 1996, respectively, and at the 1997 ISSCC as well. After being general manager for the development of various embedded memories, eSRAM, eDRAM, eFlash, eFeRAM, and eReRAM for system LSI in Panasonic, he moved to Fukuoka Institute of Technology and has been a professor since 2005. His current interests are focused on variation- tolerant memory circuit designs for the nanometer era. He holds 212 Patents including 87 U.S. Patents and has presented more than 70 journal papers and proceedings of international conferences including 10 for ISSCC and 11 for Symposium on VLSI Circuits. Dr. Yamauchi received the 1996 Remarkable Invention Award from Science and Technology Agency of Japanese Government, and the highest ISOCC2008 and IEEK ISOCC2013 Best Paper Award. 